\title{
Performance Analysis of Radio over Fiber System with Ook Based Dwdm for Fiber to Home Network
}

\author{
Ali Hussein Radhi ${ }^{1}$, Arvind Kumar Jaiswal ${ }^{2}$, Mukesh Kumar $^{3}$ \\ ${ }^{1}$ M.Tech. in optical fibre Communication Engineering in Department of Electronics and Communication \\ Engineering in SHIATS, Allahabad, India. \\ ${ }^{2}$ Prof. and Head of ECE Dept at SHIATS-Allahabad, India. \\ ${ }^{3}$ Asst. Prof. in the Department of Electronics \& Communication Engineering in SHIATS, Allahabad, India.
}

\begin{abstract}
In the recent trends in optical communication to overcome one of the major problems in the new generation of radio over fiber networks that requires being concentrated, is decreasing the quantity of bit error rate in the lowest power penalty. This paper also evaluates the results of down-link $5 \mathrm{Gbps} O O K$ signal of five radio base stations over $60 \mathrm{~km}$ dense wavelength division multiplexing single mode fiber. The BER performance of our scheme shows that our scheme is a practical solution to meet the data rate and cost-efficient of the optical links simultaneously in tomorrow's ROF access networks. This amount of bit error rate can be supposed as a negligible value in the performance of radio over fiber system for multimedia and real time applications.
\end{abstract}

Key words: $R O F$ system, DWDM, OOK signal, BER.

\section{Introduction}

There are several optical techniques for generating and transporting microwave signals over fiber. By considering the frequency of the RF signal fed into the RoF link at the head end in comparison with the signal generated at the RAU the RoF techniques may be classified into three categories - namely RF-over-fiber (RFoF), IF-over-Fiber (IFoF), or baseband-over-Fiber (BBoF) [1]. RFoF involves the transmission of the actual RF signal over the fiber. The use of Wavelength Division Multiplexing (WDM) for the distribution of RoF signals has gained importance recently. WDM enables the efficient exploitation of the fiber network's bandwidth. However, the transmission of RFoF signals is seen as inefficient in terms of spectrum utilization, since the modulation bandwidth is always small fraction of the carrier signal frequency. Radio over fiber (ROF) combines two technologies, radio frequency (RF) and optical light-wave. The advantages of ROF system is low attenuation loss (about 0.2 [2] to $0.3 \mathrm{~dB} / \mathrm{km}$ [3]), high-speed bandwidth(approximately 1Tbps [4]), protection from radio frequency interference, simple installation and low system maintenance, reduced power with reduced equipment's, supporting of multi-operator with multi- service operation, dynamic resource allocation based on the wavelength division multiplexing modulation and providing privacy and security. On the other hand, the wireless technology provides mobility characteristics for the great number of mobile users.

The utilization of the enormous bandwidth offered by optical fibre is severely hampered by the limitation in bandwidth of electronic systems, which are the primary sources and receivers of transmission data. This problem is referred to as the "electronic bottleneck". The solution around the electronic bottleneck lies in effective multiplexing. DWDM technique mentioned above are used in digital optical systems. In analogue optical systems including RoF technology, Sub-Carrier Multiplexing (SCM) is used to increase optical fibre bandwidth utilization. In SCM, several microwave subcarriers, which are modulated with digital or analogue data, are combined and used to modulate the optical signal, which is then carried on a single fibre [5], [6]. This makes RoF systems cost-effective.

The indoor wireless optical communications was invented in 1979 by Gfeller and Bapst [7]. The first idea of radio over fiber was in1990 that was used for cordless or mobile telephone service [8]. It was offered to use the diffuse emissions in the infrared band for indoor communications [9]. The wavelength division multiplexing systems have been developed in the early 1990s. They contain numerous of various wavelength channels to support the total throughput, even if it arrives nearly to 1Tbps [10].The radio systems address the user sides or base stations by providing mobility in down-link and up-link. The radio users can utilize wireless, digital cellular communication (e.g. GSM),microwave or any different type of radio signal. However, each of mentioned signals would need specific optical modulators and detectors according to the range of radio signal frequency.

\subsection{Radio-over-Fiber Technology}

\section{System Design Model}

Radio-over-Fibre (RoF) technology entails the use of optical fibre links to distribute RF signals from a central location (headend) to Remote Antenna Units (RAUs). In narrowband communication systems and 
WLANs, RF signal processing functions such as frequency up-conversion, carrier modulation, and multiplexing, are performed at the BS or the RAP, and immediately fed into the antenna. RoF makes it possible to centralize the RF signal processing functions in one shared location (headend), and then to use optical fiber, which offers low signal loss $(0.3 \mathrm{~dB} / \mathrm{km}$ for $1550 \mathrm{~nm}$, and $0.5 \mathrm{~dB} / \mathrm{km}$ for $1310 \mathrm{~nm}$ wavelengths $)$ to distribute the RF signals to the RAUs, as shown in Figure 1.

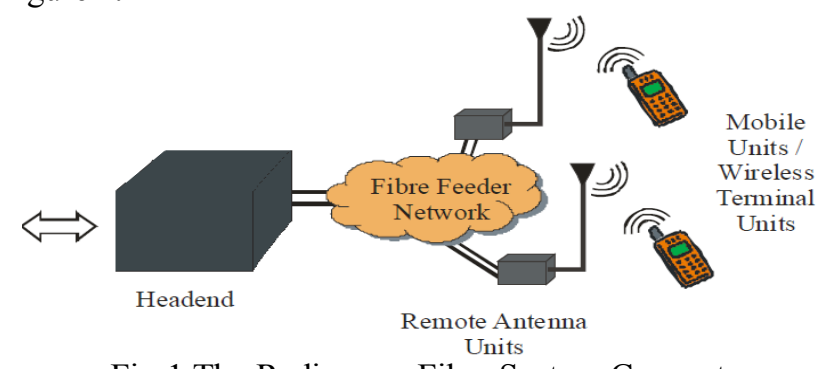

Fig:1 The Radio over Fiber System Concept

Fig. 2 indicates a simple WDM technology between multiplexer and de-multiplexer where each sender and it receiver uses a specific wavelength along an optical fiber. Dense wavelength division multiplexing furthers the transmission capacity of trunk lines [11]. In general, the modern high-speed DWDM technologies have been built of many transmission spans. It is composed of an erbium doped fiber amplifier, a single-mode fiber transmission section and a part of dispersion compensating fiber or achirped fiber bragg grating (FBG) [12]. DWDM supports 144 wavelengths with $2 \mathrm{~nm}$ spacing. It allows multiple wavelengths to be combined into one optical signal and increases the total data rate on one fiber to $1012 \mathrm{bps}[4,13]$.

This work models five radio base-stations that receive $5 \mathrm{Gbps}$ down-link on-off keying (OOK) radio signal from main control office. The main control office transmits data for the each mentioned radio base-station by dense wavelength division multiplexing at the same time over $60 \mathrm{~km}$ single mode optical fiber. This modeling also uses the direct optical modulator with power $-10 \mathrm{dBm}$ at reference range wavelength of $1500 \mathrm{~nm}$ to $1508 \mathrm{~nm}$ as electro-optic component for converting radio signal to optical light-wave power in main control office. Those optical powers will be retransferred into radio electrical signal by PIN photo-detector as optoelectrical device after traveling through optical fiber by internal total reflection law. This procedure is for transforming optical carriers to electrical current based on the corresponding wavelength in radio base-stations. the important and main parameters which are used for the OOK radio signal over $60 \mathrm{~km}$ SMF optical fiber.

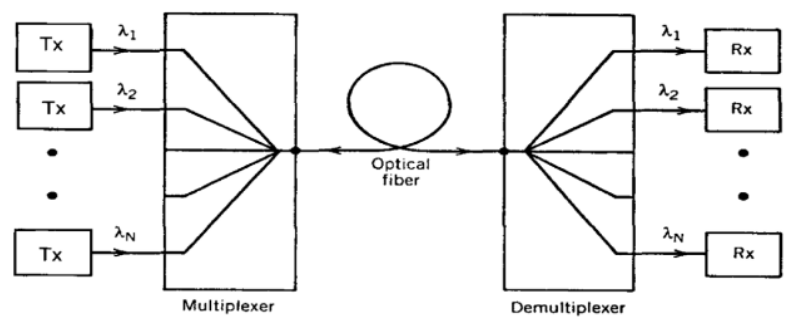

Figure 2.Wavelengths multiplexing technique [11].

\section{Simulation Results}

Reliable and comfortable radio networks are demanded in the modern communication system. Radio over fiber system indicates an advanced technology for transmission data. The radio signal has been sent by mobile user, would be modulated and transmitted over an optical fiber channel in light-wave styles. This is the result of the low attenuation loss and great bit-rate of optical fiber and also radio mobility [12].

The spectrum of the separated optical carriers is shown in Fig.3. The generated 5Gbps OOK radio signals in main control office concurrently send in down-link status for the radio base-stations over single mode fiber by DWDM technique. The main control office defines five different wavelength channels in DWDM technique for each radio base-station. DWDM supports these channels by spacing of $2 \mathrm{~nm}$ for avoidance of any interference during transmission data. The peak of optical power also occurs at reference wavelengths that have been defined in single mode fiber and DWDM. Then, down-link 5Gbps OOK radio signals are transmitted just over $60 \mathrm{~km}$ DWDM single mode fiber link without any interference at large bit rate. Based on the Fig. 3 the five stations would transmit radio signal by unique wavelength. They work on $1500 \mathrm{~nm}(\mathrm{CH} 1$ for first radio transmitter) to $1508 \mathrm{~nm}$ (CH5 for fifth radio transmitter) by spacing channels of $2 \mathrm{~nm}$. Furthermore, Fig.4 compares the measure of bit error rate (BER) sensitivity versus total received optical power between back-toback and $60 \mathrm{~km}$ length of single mode fiber by DWDM technique in the mentioned down-link radio over fiber system for all transmission channels. The attenuation loss of fiber is assumed to be $0.2 \mathrm{~dB} / \mathrm{km}$ in optical transmission link. According to the outcomes from Fig.4, the penalty of total received optical power after $60 \mathrm{~km}$ 
DWDM single mode fiber is about $0.2 \mathrm{~dB}$. For instance, the receiver sensitivity power at BER of $10^{-10}$ for the $60 \mathrm{~km}$ DWDM fiber link is $0.2 \mathrm{~dB}$ more than back-to-back. This value of power penalty can be neglected in the down-link $5 \mathrm{Gbps}$ OOK radio signal over optical fiber. This optical power difference is almost constant for all BER quantity and best performance of simulated system would be occurred at BER of $10^{-12}$. It can be seen that based on the mentioned outcomes in this paper and others previous works, radio over fiber helps to develop the reliable real-time next generation networks by significant performance and efficiency.

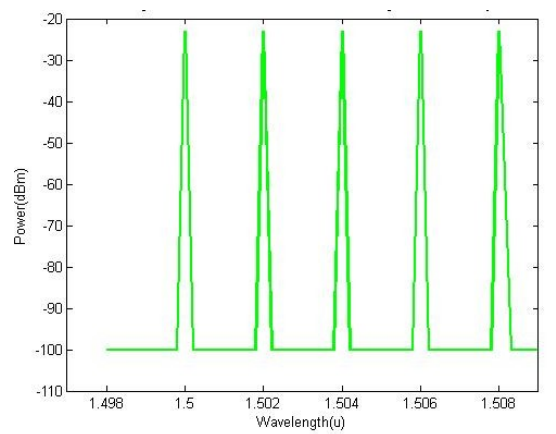

Figure 3: Down-link OOK radio signal in five base-stations over $60 \mathrm{~km}$ single mode fiber by DWDM technique

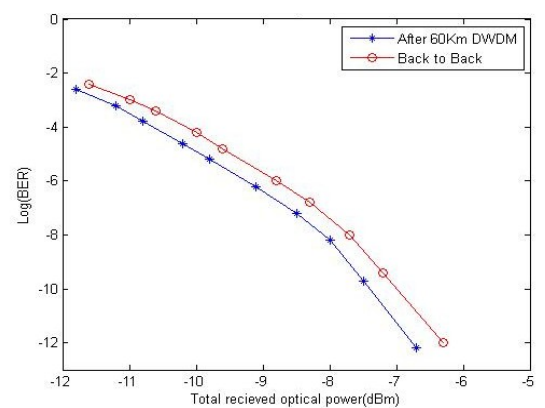

Figure 4: The measure BER of 5Gbps down-link OOK radio signal over $60 \mathrm{~km}$ DWDM single mode fiber versus total received optical power for all transmission channels

\section{Conclusion}

This paper has studied that dense wavelength division multiplexing is an applicable option (channel spacing $2 \mathrm{~nm}$ ) for developing backbone of radio over fiber network. The BER performance of our scheme shows that our scheme is a practical solution to meet the data rate and cost-efficient of the optical links simultaneously in tomorrow's ROF access networks. It can be concluded that OOK radio signal over DWDM single mode optical fiber would be a suitable solution for the multimedia and real time.

\section{References}

[1]. D. Novak, "Fiber Optics in Wireless Applications", OFC 2004 Short Course 217, 2004.

[2] H. Al-Raweshidy, Radio Over Fiber Technologies for Mobile Communications Networks: Artech house Inc., 2002.

[3] A. Ng'oma, "Radio-over-fiber technology for broadband wireless communication systems," Eindhoven,The Netherlands, PhD Thesis $9038617232,2005$.

[4] G. P. Agrawal, Fiber-Optic Communication Systems: A John Wiley \& Sons Inc., 2002.

[5]. D. Wake, S. Dupont, J-P. Vilcot, and A. J. Seeds, "32-QAM Radio Transmission Over Multimode Fibre Beyond the Fibre Bandwidth", in Proceedings of the IEEE International Topical Meeting on Microwave Photonics (MWP'01), 2001.

[6]. D. Wake, S. Dupont, C. Lethien, J-P. Vilcot, and D. Decoster, "Radiofrequency Transmission over Multimode Fibre for Distributed Antenna System Applications", Electronic Letters, Vol. 37, No. 17, pp 1087-1089(2001).

[7] A. J. Cooper, "Fiber/Radio for the provision of cordless/mobile telephony services in the access," Electronic Letter, vol. 26, pp. 2054-2056, Nov 1990.

[8] S. Hranilovic, Wireless Optical Communication Systems: Springer. 2005.

[9] F. R. Gfeller and U. Bapst, "Wireless in-house communication via diffuse infrared radiation," Proceedings of the IEEE, vol. 67, pp. 1474-1486, November 1979.

[10] C. H. Lee, Microwave Photonics (Optical Science and Engineering Series): CRC Press. 2006.

[11] K. Kitayama, et al."Dispersion effects of FBG filter and optical SSB filtering in DWDM millimeter-wave fiber-radio systems," Light wave Technology, vol. 20, pp. 1397-407, 2002.

[12] M. Marciniak, "Application of Radio over Fiber Technology to Enable Converged Optical and Wireless Next Generation Networking," in Second International Conference on Access Networks \& Workshops, Access Nets '07., 2007, pp. 1-7.

[13] J. R. Vacca, Optical Networking Best Practices Handbook: A John Wiley \& Sons Inc., 2007. 


\section{AUTHOR'S PROFILE}

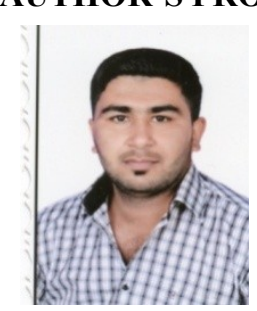

\section{Ali Hussein Radhi}

Received his bachelor of communication engineering from Technical College Najaf in IRAQ. M.Tech. in optical fibre Communication Engineering in Department of Electronics and Communication Engineering in SHIATS, Allahabad in India. Email: ali_hussein8944@yahoo.com

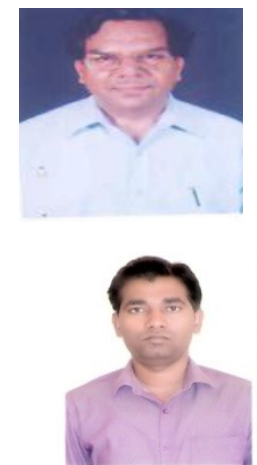

A.K. Jaiswal is Prof. and Head of ECE Dept at SHIATS-Allahabad. He Obtained M.Sc. in Tech. Electronic \& Radio Engg. from Allahabad University in1967. He guided various projects \& research at undergraduate \&postgraduate level. He has more than 35years Industrial, research \& Teaching experience and actively involved in research and publications. His area of interest includes Optical Networks and satellite communication.

Mukesh Kumar is working as a Asst. Prof. in the Department of Electronics \& Communication Engineering in SHIATS, Allahabad. He received his M.Tech. Degree in Advanced Communication System Engineering from SHIATS, Allahabad in 2010. His research is focused on Signal processing, Wireless Sensor Network and Computer Networks ,Microwave Engineering, as well as Optical fiber communication. 